\title{
Evaluating the proposal of paediatric virology: An interview with Professor Tina Dalianis, Professor of Tumour Virology at Karolinska Institutet
}

\author{
IOANNIS N. MAMMAS and DEMETRIOS A. SPANDIDOS \\ Department of Clinical Virology, School of Medicine, University of Crete, Heraklion 71003, Greece
}

Received June 22, 2018; Accepted July 23, 2018

DOI: $10.3892 / \mathrm{etm} .2018 .6543$

\begin{abstract}
Professor Tina Dalianis, Professor of Tumour Virology at Karolinska Institutet in Stockholm, Sweden, is a well-known virologist with a strong international reputation and seminal contributions in tumour virology in the fields of human papillomaviruses (HPVs) and polyomaviruses. According to Professor Dalianis, over the past decades virology has significantly contributed to the understanding of many infectious diseases in medicine. In addition, quite a few antiviral therapies have been developed in recent years that have been very helpful, while the study of viruses has also provided valuable information about their host cells and basic cell biology. She highlights the association of HPVs with tonsillar and base of tongue cancers, as well as their epidemic rise. She also refers to additional biomarkers, which are under investigation, in order to better individualise patient care, by for example, de-escalating or targeted therapy, whenever this is possible. She clarifies that the current prophylactic vaccines against HPVs are very efficient for the types they are directed against and she hopes that boys will also soon be vaccinated against HPVs, particularly in countries where there is a low percentage of girls being vaccinated. According to Professor Dalianis, the knowledge of virology is very useful in general paediatric practice; the proposal of paediatric virology as a medical subspecialty is absolutely correct and it would be very useful, as there is much to be done. In the context of the '4th Workshop on Paediatric Virology', Professor Tina Dalianis will give the plenary lecture of this scientific event and will focus on changes in the prevalence of different HPVs in genital and oral samples in some groups of youth in Sweden during the introduction of the vaccines against HPVs.
\end{abstract}

Correspondence to: Professor Demetrios A. Spandidos, Department of Clinical Virology, School of Medicine, University of Crete, Heraklion 71003, Greece

E-mail: spandidos@spandidos.gr

Key words: virology, tumour virology, paediatric virology, HPVs, workshop, Professor Tina Dalianis, Karolinska Institutet

\author{
Contents \\ 1. Introduction \\ 2. Questions and Answers
}

\section{Introduction}

Professor Tina Dalianis (Fig. 1), Professor of Tumour Virology at the Department of Oncology-Pathology at Karolinska Institutet in Sweden, was born on November 1951 in Athens, Greece and she spent her childhood in Greece, England and Sweden. She studied medicine at the Karolinska Institutet from 1968 to 1974 and she completed her $\mathrm{PhD}$ in tumour biology under the supervision of Professor George Klein in 1980 (1). From 1986 to 1991, she attended the specialist training programme in clinical virology at the Stockholm City Council Microbiological Central Laboratory in Sweden, with a twoyear break between 1987 and 1989, to do a postdoc in the UK in Professor Peter Rigby's laboratory at the National Institute for Medical Research in London. After completing her postdoc and specialist training in clinical virology, in 1992, she moved to the Department of Clinical Immunology at the Huddinge University Hospital. From 1995 to 2002, she was appointed as consultant and head of the Transplantation Immunology Section at the Karolinska University Hospital and in 1995, she was elected as lecturer at the Division of Clinical Virology in the same hospital. In 2000, she was appointed as Professor of Tumour Virology at the Department of Oncology-Pathology at Karolinska Institutet, and since then, she heads the tumour virology group. From 2002 to 2011, she was head of the Department of Oncology-Pathology at the Karolinska Institutet, a 6 year rotation position, which she held for 9 years.

One of Professor Dalianis's earliest most important achievements was, in 1989 , the detection that it was possible to vaccinate against tumour outgrowth with synthetic peptides. Following her return from London, she studied the role of human papilloma viruses (HPVs) in head and neck cancer. In 2000, her group was the first to show the association between HPVs and tonsillar cancer, and later in 2004, for the base of tongue cancer and that the detection of HPVs was a prognostic favourable factor. Her group was also the first to show that the epidemic increase of tonsillar and base of tongue cancer was due to an increase in the number of HPV-positive cases. 
Recently, her group identified specific biomarkers that together with the HPV-positive status, can predict an even better prognosis in HPV-positive and tonsillar and base of tongue cancer, and some of these markers are targetable.

Professor Dalianis is a well-known tumour virologist with a strong international reputation and has made seminal contributions in tumour virology in the fields of HPVs and polyomaviruses (2-14). She is an author of more than 200 scientific contributions and has a citation index of over 5,000 and an h-index of 35. To date, she has helped and supported the Paediatric Virology Study Group (PVSG) and has been member of its academic advisory board. In September 22nd, 2018, Professor Dalianis will chair the "4th Workshop on Paediatric Virology' and her plenary lecture is entitled 'Changes in HPVs prevalence in genital and oral samples in some groups of youth in Sweden during the introduction of the HPV vaccines'. In the context of this scientific event, she will also receive the '2018 Paediatric Virology Award in Virology' for her outstanding academic, research and publishing contribution to tumour virology.

\section{Questions and Answers}

Question: Dear Professor Tina Dalianis, first of all, thank you for your acceptance to chair our "4th Workshop on Paediatric Virology' and give the plenary lecture of this scientific event. Your lecture will focus on changes in the prevalence of HPVs in genital and oral samples in some groups of youth in Sweden during the introduction of the vaccines against HPVs. According to your catch-up vaccination virology data, how effective are the current HPV vaccines?

Answer: The current HPV vaccines are very efficient prophylactic vaccines for the types they are directed against. They protect against infection against these types of viruses and this way against the cancers that these HPVs can cause.

Question: In the context of last year's workshop, Nobelist Professor Harald zur Hausen stated that 'vaccination of boys against HPVs should be included in the national vaccination programmes at the same age as girls' and that 'vaccination of boys should be mandatory' $(15,16)$. One of the 10 top key messages of this workshop was that 'HPV vaccine uptake should be promoted in countries with low HPV vaccination rates to reduce the cervical and anogenital cancer burden, as well as for the prevention of genital warts; inclusion of males in childhood HPV immunisation programmes should be considered' (17). What are the current recommendations in Sweden? What are the participation rates among female adolescents into the programme against HPVs in Sweden?

Answer: The tetravalent Gardasil vaccine is currently given to girls aged 10-12 years within the school based vaccination programme and approximately $80 \%$ of the girls are vaccinated today. In addition, many counties in Sweden give catch-up vaccination to girls/women up to the age of 20 or 26 years of age, depending on the county. The Public Health Agency of Sweden has recommended HPV vaccinations for boys aged 10-12 years within the school based vaccination programme, but the government has not yet said go ahead. Personally, I agree with Professor Harald zur Hausen and hope that also boys soon will be vaccinated. In countries where there is a low percentage

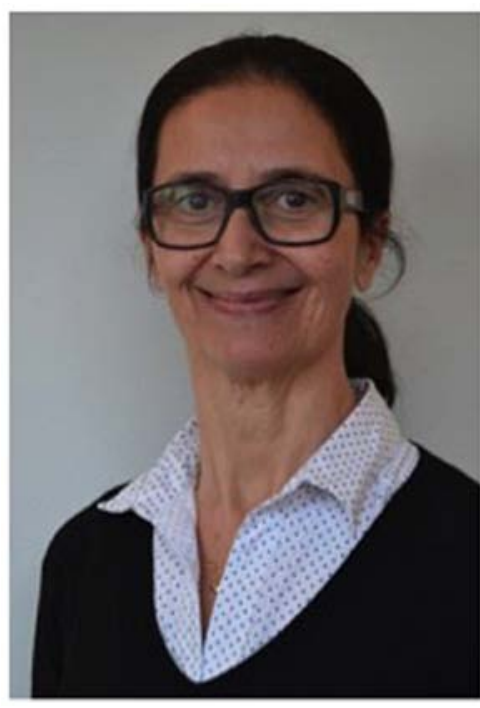

Figure 1. Professor Tina Dalianis, Professor of Tumour Virology at the Department of Oncology-Pathology at Karolinska Institutet in Sweden and Chair of the '4th Workshop on Paediatric Virology', Athens, Greece.

of girls being vaccinated, as for example, in Denmark with only $40 \%$ of the girls being vaccinated, there it is even more important that boys are vaccinated in order to also achieve herd immunity.

Question: Your contribution to oral HPV virology and the 'ongoing epidemic' of oropharyngeal cancer has been recognised by our scientific community. Recently, the January 2018 issue of the International Journal of Oncology was dedicated to you. Among your achievements on this field, which ones do you consider as the most significant?

Answer: I am not really sure. I believe the fact that we disclosed the association of HPVs with tonsillar and later base of tongue cancer and that this cancer had better outcome than HPV-negative tonsillar and base of tongue cancer was a major contribution. We were also among the very first showing that the epidemic rise of tonsillar and base of tongue cancer was due to the HPVs-positive cases and I suppose this was a major contribution too. Now, we are aiming to find additional biomarkers in order to individualise patient care in this growing group of patients, by for example, de-escalating or targeting therapy, whenever this is possible.

Question: Can you explain to us what biomarkers are you studying at the moment? How these biomarkers could be used to individualise patient care? We have read with real interest your last review article entitled 'Human papillomavirus and potentially relevant biomarkers in tonsillar and base of tongue squamous cell carcinoma' published last year in Anticancer Research (2).

Answer: In the context of tonsillar and base of tongue cancer, a biomarker can be the size of the tumour, the age of the patient and these we would define as clinical biomarkers. Then, we have other types of biomarkers, more molecular or cellular ones: e.g. having high $\mathrm{CD}^{+}$tumour infiltrating lymphocyte (TIL) counts is also a positive biomarker. Other biomarkers are the presence of HPV16 E2 mRNA expression. 
Question: Research on HPVs has been expected to end-up after the introduction of the HPV vaccines into clinical practice. However, their introduction has led to an increase in research on HPVs. What remains to be done? Is there any place for a next Nobel notification on the field of HPVs virology?

Answer: There is always a lot to be done. It will take decades before these tumours decrease or even disappear. It may not warrant a Nobel Prize, however. Many patients still suffer from cervical cancer and other HPV-associated cancers and of course one would like to help every patient as much as possible with all means possible. If immunotherapy or immunoregulatory therapy were possible of course this would be helpful. There are also new drugs on the market with targeted therapy for other cancer types, so just like for the other cancer types one needs to find out what can be used for these patients. There is no time to relax yet and it is important to keep on working hard.

Question: You are considered as a leading expert worldwide on virology with a strong international reputation. What are the principles that helped you to this successful career as a medical student, as a medical doctor as well as a principal researcher in virology?

Answer: I have been interested in research since I was 10-12 years of age after reading Madame Curies' short book about her mother, when waiting for a piano lesson. I started in medical school not yet 17 years old with the aim to do research and when I was 18 years I started in Professor George Klein's laboratory in parallel to my medical studies. This laboratory was one of the most exciting laboratories at the time with an extremely international environment. In the beginning, I understood extremely little and often felt very insufficient, but I did not want to give up and with time, I understood more and more, and have enjoyed science more and more. It is so fascinating with new questions and with time seeing some of these questions being answered.

Question: The 'father of HPV virology', Professor Harald zur Hausen, had to wait for several years in order to be accepted by the worldwide scientific community. His findings at the beginning of the 1970's, in a time when the false dogma implicating herpes simplex viruses (HSVs) in the aetiology of cervical cancer had gained a catholic scientific acceptance, were managed with scepticism. His hypothesis that cervical cancer is mainly caused by HPVs was completely novel and the scientific community at that time was negative to accept the viral aetiology of cervical cancer. For this reason, it took many years for the HPV vaccines to be included into clinical practice. According to your opinion, why did this happen?

Answer: This often happens. As you know, many times people claim that a virus causes this or that, for example, the HSV theory was wrong. So, there is a natural scepticism that is healthy, but unfortunately, this can also delay the acceptance of correct findings. We had the same situation with no one believing that HPVs had anything to do with tonsillar and base of tongue cancer. I have met Professor Harald zur Hausen many times and was surprised when he told me about all this above, as well as there was no interest in a vaccine either in the beginning, which is even more puzzling and not understandable at all. In Sweden, in 2008, when Professor zur Hausen received the Nobel Prize, they still did not believe us at the Karolinska
Institute. However, in his Nobel Lecture Professor zur Hausen gave us support and that helped!

Question: George N. Papanicolaou (Kyme, Island of Euboea, Greece, 1883 - Miami, Florida, USA, 1962) was an exceptional human, scientist and academic teacher, whose major scientific work was performed at the Cornell University Medical College in New York, USA. We would like your comment on the contribution of George N. Papanicolaou in modern medicine. Answer: This has been a fantastic contribution. For its time it was a great test. Its accuracy is not so safe really since it does not catch all pre-stages, but the fact that one can repeat the test and that it takes time to develop cervical cancer, repeating it regularly has made it possible to catch very many pre-stages and save very many lives.

Question: Over the past two decades, the Department of Clinical Virology at the University of Crete School of Medicine has also contributed to HPV research. Our laboratory was the first in Greece, where the polymerase chain reaction (PCR) technique was performed for the detection of HPVs. Moreover, our laboratory was among the few laboratory worldwide, where paediatric oral and skin samples were studied in relation to their HPV status. How do you evaluate this contribution?

Answer: It is always great being early in accepting new findings and bringing in new techniques. The Crete School of Medicine has a very good international reputation. This helps the population in general and one can many times see that things go better in areas, where there is a hub of a very good hospital, with good staff and scientists, that is proactive and foreseeing.

Question: You were born in Athens and you spent part of your childhood in Greece. Since 2010, Greece is under a continuous financial crisis, which also affects the health of its citizens. Unfortunately, this period of time is also characterised by the 'brain drain' of young Greek scientists, including scientists, who have studied medicine and basic sciences in Greece.

Answer: What has happened is most unfortunate. Just before 2008, all was going well for Greece and many scientists were optimistic and returning to Greece to start laboratories there. I have met quite a few. What happened after 2008 was to me personally, quite a shock, and nothing I expected. I believe this, too, was unexpected for most of us. However, one should remember that Greece has progressed immensely over the past decades and the general knowledge of its youth and also its infrastructure is very good. If the economy changes, I do not believe one needs to start from nothing. The young people in Greece are much more educated than their parents and grandparents and should make rapid progress. Then, again one never knows what lies ahead for us in the future.

Question: Your whole scientific work has been performed in one of the most significant institutions in the world, Karolinska Institutet in Sweden. How important was this for your scientific efforts?

Answer: As I mentioned above, being in the laboratory of George Klein was most likely the most important thing in my scientific career. George Klein was a humanist, who had 
survived the Second World War and managed to flee from being sent to the concentration camps. He had a wish to make science available to as many people, who wanted to contribute as possible. He shared all he knew with all of us and the environment was extremely creative. George Klein and all the people in the laboratory that came from all over the world made a huge impact on me and the way I continued my career, although I believe I had a personal drive even before that.

Question: During your scientific career, did you try to return to Greece? Did our local Medical Schools tried to persuade you to return?

Answer: I was four when we left Greece for England, and I started primary school there. Then my parents moved to Sweden when I was 8 years old. Then I started reading medicine as I mentioned above in order to do science. In the 1970s, when I was approximately 20 years old, I spent a summer at 'Theagenion' in Thessaloniki, at the laboratory of Professor Joyce Taylor Papdimitriou. I realised it was a tough time and they worked hard and had to go to the butcher to get foetal calf serum for their cell cultures. I understood that although the work was good, it was very hard to do science in Greece, because one had so much less resources. Even at Klein's laboratory, the resources were not extensive and it was difficult too. So, I decided if I was going to do science I could not go back to Greece. However, I have always been interested in Greece, and somehow wanted to contribute if I could. I have therefore tried to help Greek students and I have had a Greek PhD student, too. I was also hoping to collaborate with Greek laboratories, and was lucky to do so through a joint EU collaboration at the Hellenic Pasteur Institute in Athens for some years in the early mid-2000s.

Question: 'As more than $90 \%$ of the cases attended to in a paediatric unit are children with a viral infection, knowledge of virology is important in paediatrics'. Please share your view on this statement.

Answer: You are very right. This is especially obvious in November to March in Sweden, when almost every second child has a viral infection. Of course knowledge of virology would be very helpful in general paediatric practice and also if one had rapid viral diagnostic tests in order to help patients and their parents, and lower anxiety, since in most cases most infections go away. It is also possible we will get more viral vaccines and anti-virals, but we are not there yet.

Question: Paediatric virology is indeed an exciting paediatric field exploring new knowledge of neonatal and paediatric viral infections and how to detect, manage, and treat them. According to your scientific background as a virologist, what advances during the last years do you consider as the most significant in the field of neonatal and paediatric viral infections?

Answer: There are many fantastic achievements. However, I must admit that I have not been working within this field for decades. An old contribution is the rubella vaccine. A newer contribution is the HepB vaccine for one. However, also the possibility to treat early HSV infections so that they do not become neonatal at delivery is also important. Furthermore, there are new anti-hepatitis drugs, but I do not have the knowledge of their use in the neonatal period. An hepatitis $\mathrm{C}$ virus (HCV) and a cytomegalovirus (CMV) vaccine might also be great contributions later on in the future. I am sure there is plenty more to be done.

Question: Paediatric virology has recently been proposed as a new candidate of paediatric subspecialisation (18). Both paediatric clinicians and basic scientists have been proposed to possess a critical educational role on this. I would appreciate if I could have your opinion on the future role of paediatric virology as a new paediatric subspecialty.

Answer: I read through your very interesting proposal and I have to admit that I have not thought of this before. You are absolutely correct in that it would be very helpful and there is extremely much to do. I do not practise virology any more in the clinic; however, for a long time (1986-1999), I had my research there and had contact frequently, although between 1992 and 1999, my clinical work was in immunology and I was head of the transplant section at the clinical ward. However, one of my close friends and former colleague and $\mathrm{PhD}$ student, Ilona Lewensohn Fuchs is still active in virology and head of the Virology Clinic of the south part of the Karolinska University Hospital at Huddinge. She often informs me of what is going on. In Stockholm, some virologists are especially interested in neonatal viral infections and meet and collaborate with gynaecologists and paediatricians. However, there is no paediatric virology speciality. If I understand from you, you would like a paediatrician to learn more of virology, rather than the opposite. Here, both specialities try to get closer to each other to solve joint problems. In any case whatever one does, it is an important subject worth pursuing and who ever does is doing something important.

Question: In our last workshop, we discussed the creation of an educational e-platform on paediatric virology aiming to the medical education of paediatric trainees and junior paediatricians on viral neonatal and paediatric infections (17).

Answer: I think it seems like a very good idea. However, I believe it is also important to have local knowledge of colleagues, gynaecologists' virologists, who are especially interested in paediatric infections, or prenatal infections and that this group possibly meets and keeps in touch and that their knowhow is available to other colleagues.

Question: This year, 100 years have passed from the 'Spanish' influenza pandemic in 1918, and I would like your comment on this anniversary. In 1918, one third of the population of the Greek island of Skyros, close to the island of Euboea, died of this outbreak (19). How vulnerable are we indeed for viral-induced epidemic threats in the future?

Answer: Pandemics can become very dangerous, especially since we travel as much as we do. They can be very contagious and very dangerous, or very contagious but not so dangerous, or not so contagious and dangerous, but not immediately. We have however, not to forget more recently had the human immunodeficiency virus (HIV) epidemic, which has been of another kind and killed very many people.

Question: On behalf of the PVSG, it is a great honour to announce you that you will be the recipient of the 
'2018 Paediatric Virology Award in Virology' for your outstanding academic, research and publishing contribution to tumour virology. Could we have your statement on the value of virology in medicine and modern science and its educational and research perspectives?

Answer: I want to thank you for this honour to me. During the past decades virology has significantly contributed to explaining many infectious diseases in medicine and been extremely useful for the understanding of many diseases. In addition, quite a few antiviral therapies have been developed in recent years that have been very helpful. Finally, the study of viruses has also provided valuable information about their host cells, basic cell biology, and molecular biology.

Question: Thank you for your answers and your valuable time; we are looking forward to your chairmanship and your plenary lecture in Athens on September.

\section{References}

1. Dalianis T: Selection and characterization of antigenic variants from tumor cells ( $\mathrm{PhD}$ thesis). Karolinska Institutet, Stockholm, 1980.

2. Näsman A, Bersani C, Lindquist $\mathrm{D}, \mathrm{Du} \mathrm{J}$, Ramqvist $\mathrm{T}$ and Dalianis T: Human papillomavirus and potentially relevant biomarkers in tonsillar and base of tongue squamous cell carcinoma. Anticancer Res 37: 5319-5328, 2017.

3. Bersani C, Sivars L, Haeggblom L, DiLorenzo S, Mints M, Ährlund-Richter A, Tertipis N, Munck-Wikland E, Näsman A, Ramqvist $\mathrm{T}$, et al: Targeted sequencing of tonsillar and base of tongue cancer and human papillomavirus positive unknown primary of the head and neck reveals prognostic effects of mutated FGFR3. Oncotarget 8: 35339-35350, 2017.

4. Bersani C, Mints M, Tertipis N, Haeggblom L, Sivars L, Ährlund-Richter A, Vlastos A, Smedberg C, Grün N, Munck-Wikland E, et al: A model using concomitant markers for predicting outcome in human papillomavirus positive oropharyngeal cancer. Oral Oncol 68: 53-59, 2017.

5. Haeggblom L, Nordfors C, Tertipis N, Bersani C, Ramqvist T, Näsman A and Dalianis T: Effects of irradiation on human leukocyte antigen class I expression in human papillomavirus positive and negative base of tongue and mobile tongue squamous cell carcinoma cell lines. Int J Oncol 50: 1423-1433, 2017.

6. Sivars L, Landin D, Grün N, Vlastos A, Marklund L, Nordemar S, Ramqvist T, Munck-Wikland E, Näsman A and Dalianis T: Validation of Human Papillomavirus as a Favourable Prognostic Marker and Analysis of CD8+ Tumour-infiltrating Lymphocytes and Other Biomarkers in Cancer of Unknown Primary in the Head and Neck Region. Anticancer Res 37: 665-673, 2017.

7. Sivars L, Bersani C, Grün N, Ramqvist T, Munck-Wikland E, Von Buchwald C and Dalianis T: Human papillomavirus is a favourable prognostic factor in cancer of unknown primary in the head and neck region and in hypopharyngeal cancer. Mol Clin Oncol 5: 671-674, 2016.
8. Sivars L, Landin D, Haeggblom L, Tertipis N, Grün N, Bersani C, Marklund L, Ghaderi M, Näsman A, Ramqvist T, et al: Human papillomavirus DNA detection in fine-needle aspirates as indicator of human papillomavirus-positive oropharyngeal squamous cell carcinoma: A prospective study. Head Neck 39: 419-426, 2017.

9. Chaturvedi AK, Song H, Rosenberg PS, Ramqvist T, Anderson WF, Munck-Wikland E, Ye W and Dalianis T: Tonsillectomy and Incidence of Oropharyngeal Cancers. Cancer Epidemiol Biomarkers Prev 25: 944-950, 2016.

10. Sivars L, Tani E, Näsman A, Ramqvist T, Munck-Wikland E and Dalianis T: Human Papillomavirus as a Diagnostic and Prognostic Tool in Cancer of Unknown Primary in the Head and Neck Region. Anticancer Res 36: 487-493, 2016.

11. Franzén J, Ramqvist T, Bogdanovic G, Grün N, Mattson J and Dalianis T: Studies of human polyomaviruses, with HPyV7, $\mathrm{BKPyV}$, and $\mathrm{JCPyV}$ present in urine of allogeneic hematopoietic stem cell transplanted patients with or without hemorrhagic cystitis. Transpl Infect Dis 18: 240-246, 2016.

12. Grandahl M, Larsson M, Dalianis T, Stenhammar C, Tydén T, Westerling $\mathrm{R}$ and Nevéus T: Catch-up HPV vaccination status of adolescents in relation to socioeconomic factors, individual beliefs and sexual behaviour. PLoS One 12: e0187193, 2017.

13. Grandahl M, Rosenblad A, Stenhammar C, Tydén T, Westerling R, Larsson M, Oscarsson M, Andrae B, Dalianis T and Nevéus T: School-based intervention for the prevention of HPV among adolescents: A cluster randomised controlled study. BMJ Open 6: e009875, 2016.

14. Grün N, Ährlund-Richter A, Franzén J, Mirzaie L, Marions L, Ramqvist $\mathrm{T}$ and Dalianis T: Follow-up on oral and cervical human papillomavirus prevalence 2013-2015 in youth at a youth clinic in Stockholm, Sweden. Infect Dis (Lond) 48: 169-170, 2016.

15. Mammas IN and Spandidos DA: Paediatric Virology as a new educational initiative: An interview with Nobelist Professor of Virology Harald zur Hausen. Exp Ther Med 14: 3329-3331, 2017.

16. Zur Hausen H, Mammas IN and Spandidos DA: HPV vaccination in boys: Determining the clinical relevance of this strategy. Exp Ther Med 14: 3327-3328, 2017.

17. Mammas IN, Greenough A, Theodoridou M, Kramvis A, Rusan M, Melidou A, Korovessi P, Papaioannou G, Papatheodoropoulou A, Koutsaftiki C, et al: Paediatric Virology and its interaction between basic science and clinical practice (Review). Int J Mol Med 41: 1165-1176, 2018.

18. Mammas IN, Greenough A, Theodoridou M and Spandidos DA: Paediatric Virology: A new paediatric subspecialty? A proposal at the Workshop on Paediatric Virology, Athens, October 10 , 2015. Exp Ther Med 11: 3-5, 2016.

19. Mammas IN, Theodoridou M and Spandidos DA: The 1918 Spanish flu outbreak that devastated a Greek island underlines past lessons that must never be forgotten. Acta Paediatr: Mar 31, 2018 (Epub ahead of print).

This work is licensed under a Creative Commons Attribution-NonCommercial-NoDerivatives 4.0 International (CC BY-NC-ND 4.0) License. 